\title{
GENETIC DISORDERS
}

\section{Epileptic Encephalopathy Due to BRAT1 Pathogenic Variants}

\author{
Siddharth Srivastava, $\mathrm{MD}^{1}$ and Sakkubai Naidu, $\mathrm{MD}^{2 *}$ \\ ${ }^{I}$ Department of Neurology, Boston Children's Hospital, Boston, MA; ${ }^{2}$ Hugo W. Moser Research Institute at Kennedy Krieger Institute, Baltimore, MA \\ *Correspondence: Dr. Sakkubai Naidu, E-mail: naidu@kennedykrieger.org
}

Related Article: Horn D, Weschke B, Knierim E, Fischer-Zirnsak B, Stenzel W, Schuelke M et al. BRAT1 mutations are associated with infantile epileptic encephalopathy, mitochondrial dysfunction, and survival into childhood. Am J Med Genet A 2016 Sep;170(9):2274-81. Keywords: Intractable Epilepsy; Microcephaly; Hypertonia; Apnea; BRAT1

Investigators from Institut für Medizinische Genetik und Humangenetik have highlighted the role of compound heterozygous BRATl variants in two German brothers with variable presentations of intractable epilepsy, poor development, postnatal microcephaly, hypertonia, apnea, and infantile/childhood death. The older brother (Pt 1) died at 5.75 years, while the younger brother ( $\mathrm{Pt} 2)$ died at 2 months. Seizure onset occurred at 5 months in Pt 1 and at birth in Pt 2 (and possibly in utero). Seizures were myoclonic, refractory to treatment, and accompanied by apnea, bradycardia (Pt 2), and focal/multifocal epileptiform discharges. Microcephaly was severe. Pt 1 achieved some turning and Pt 2 acquired no milestones. Appendicular hypertonia was present in both. Pt 2's brain MRI was normal; Pt 1's brain MRI showed corpus callosum thinning, enlarged CSF fluid spaces, and delayed myelination. Next-generation sequencing (NGS) of the disease-associated genome $(\sim 2800$ genes $)$ revealed a compound heterozygous variant in BRAT1 [c.638_639insA (p.V214fs 189*); c.1134+1G>A], confirmed in both siblings. The frameshift variant, which was maternally inherited, is a known change associated with lethal neonatal rigidity and multifocal seizure syndrome (RMFSL). The other variant, which was paternally inherited, alters splicing, evident by reduced BRAT1 mRNA expression in the father. Skeletal muscle biopsy from Pt 2 revealed myofiber immaturity, decreased cyclooxygenase staining, and decreased cytochrome c oxidase activity. [1]

COMMENTARY. This study expands the knowledge surrounding $B R A T 1$-related disorders, particularly its clinical heterogeneity. Some of the first reports of this disorder characterized it as a particularly severe, rapidly progressive, intractable epileptic encephalopathy with age of presentation at birth or shortly thereafter $[2,3]$. While these earlier investigations suggested it is lethal in the first few months of life, this present report points to increased survival into childhood (Pt 1) as one of the features of the disorder. Moreover, other manifestations in Pt 1 - later onset of epilepsy, postnatal microcephaly, and hypertonia - suggest a less affected phenotype. In fact, in addition to the severe lethal form known as RMFSL, both mild and moderate forms of BRAT1-related disorders may exist. Mildly affected individuals may present with intellectual disability without epilepsy/seizures, ataxia, cerebellar atrophy, and continued survival through late childhood [4]. Given the phenotypic differences seen with siblings, intrafamilial variability can occur.

This study also demonstrates that mitochondrial dysfunction may be a hallmark of BRAT1-related disorders. Pt 2's skeletal muscle biopsy showed evidence of impaired mitochondrial energy production. In another study, BRAT1 knockdown resulted in cells with increased glucose requirements, increased reactive oxygen species levels, and decreased ATP production [5]. Defects in mitochondrial metabolism, combined with defects in some of the other roles of BRAT1 including DNA repair and cell growth [6], may account for some of the presentations of this disorder.

Finally, this study highlights the role of NGS in diagnosing causes of epileptic encephalopathy. Depending on the laboratory, BRATl may not be one of the genes sequenced as part of an epileptic encephalopathy panel. Increased awareness of this disorder, combined with utilization of NGS, may lead to earlier diagnoses.

\section{Disclosures}

The author(s) have declared that no competing interests exist.

\section{References}

1. Horn D, Weschke B, Knierim E, Fischer-Zirnsak B, Stenzel W, Schuelke $M$ et al. BRAT1 mutations are associated with infantile epileptic encephalopathy, mitochondrial dysfunction, and survival into childhood. Am J Med Genet A 2016 Sep;170(9):2274-81. http://dx.doi.org/10.1002/ ajmg.a.37798 PMID:27282648

2. Puffenberger EG, Jinks RN, Sougnez C, Cibulskis K, Willert RA, Achilly NP et al. Genetic mapping and exome sequencing identify variants associated with five novel diseases. PLoS One 2012;7(1):e28936. http://dx.doi.org/10.1371/journal.pone.0028936 PMID:22279524

3. Saunders CJ, Miller NA, Soden SE, Dinwiddie DL, Noll A, Alnadi NA et al. Rapid whole-genome sequencing for genetic disease diagnosis in neonatal intensive care units. Sci Transl Med 2012 Oct;4(154):154ra135. http://dx.doi.org/10.1126/scitranslmed.3004041 PMID:23035047

4. Srivastava S, Olson HE, Cohen JS, Gubbels CS, Lincoln S, Davis BT et al. BRAT1 mutations present with a spectrum of clinical severity. Am J Med Genet A 2016 Sep;170(9):2265-73. http://dx.doi.org/10.1002/ ajmg.a.37783 PMID:27282546

5. So EY, Ouchi T. BRAT1 deficiency causes increased glucose metabolism and mitochondrial malfunction. BMC Cancer 2014 Jul;14(1):548. http://dx.doi.org/10.1186/1471-2407-14-548 PMID:25070371

6. Aglipay JA, Martin SA, Tawara H, Lee SW, Ouchi T. ATM activation by ionizing radiation requires BRCA1 -associated BAAT1. J Biol Chem 2006 Apr;281(14):9710-8. http://dx.doi.org/10.1074/jbc.M510332200 PMID:16452482 